\title{
The broad-line region and dust torus size of the Seyfert 1 galaxy PGC 50427^
}

\author{
F. Pozo Nuñez ${ }^{1}$, M. Ramolla ${ }^{1}$, C. Westhues ${ }^{1}$, M. Haas ${ }^{1}$, R. Chini ${ }^{1,2}$, K. Steenbrugge ${ }^{2,4}$, A. Barr Domínguez ${ }^{1,2}$, \\ L. Kaderhandt ${ }^{1}$, M. Hackstein ${ }^{1}$, W. Kollatschny ${ }^{3}$, M. Zetzl ${ }^{3}$, K. W. Hodapp ${ }^{5}$, and M. Murphy ${ }^{6}$ \\ 1 Astronomisches Institut, Ruhr-Universität Bochum, Universitätsstraße 150, 44801 Bochum, Germany \\ e-mail: francisco.pozon@gmail.com \\ 2 Instituto de Astronomia, Universidad Católica del Norte, Avenida Angamos 0610, Casilla 1280 Antofagasta, Chile \\ 3 Institut für Astrophysik, Universität Göttingen, Friedrich-Hund Platz 1, 37077 Göttingen, Germany \\ ${ }^{4}$ Department of Physics, University of Oxford, Keble Road, Oxford OX1 3RH, UK \\ 5 Institute for Astronomy, University of Hawaii, 640 North Aohoku Place, Hilo, HI 96720, USA \\ ${ }^{6}$ Departamento de Física, Universidad Católica del Norte, Avenida Angamos 0610, Casilla 1280 Antofagasta, Chile \\ Received 16 February 2015 / Accepted 19 February 2015
}

\begin{abstract}
We present the results of three-year monitoring campaigns of the $z=0.024$ type 1 active Galactic nucleus (AGN) PGC 50427. Using robotic telescopes of the Universitätssternwarte Bochum near Cerro Armazones in Chile, we monitored PGC 50427 in the optical and near-infrared (NIR). Through the use of photometric reverberation mapping with broad- and narrowband filters, we determine the size of the broad-line emitting region by measuring the time delay between the variability of the continuum and the $\mathrm{H} \alpha$ emission line. The $\mathrm{H} \alpha$ emission line responds to blue continuum variations with an average rest frame lag of $19.0 \pm 1.23$ days. Using single epoch spectroscopy obtained with the Southern African Large Telescope (SALT) we determined a broad-line H $\alpha$ velocity width of $1020 \mathrm{~km} \mathrm{~s}^{-1}$ and in combination with the rest frame lag and adoption of a geometric scaling factor $f=5.5$, we calculate a black hole mass of $M_{\mathrm{BH}} \sim 17 \times 10^{6} M_{\odot}$. Using the flux variation gradient method, we separate the host galaxy contribution from that of the AGN to calculate the rest frame $5100 \AA$ luminosity at the time of our monitoring campaign. We measured small luminosity variations in the AGN ( 10\%) accross the three years of the monitoring campaign. The rest frame lag and the host-subtracted luminosity permit us to derive the position of PGC 50427 in the BLR size - AGN luminosity diagram, which is remarkably close to the theoretically expected relation of $R \propto L^{0.5}$. The simultaneous optical and NIR ( $J$ and $K_{\mathrm{s}}$ ) observations allow us to determine the size of the dust torus through the use of dust reverberation mapping method. We find that the hot dust emission $(\sim 1800 \mathrm{~K})$ lags the optical variations with an average rest frame lag of $46.2 \pm 2.60$ days. The dust reverberation radius and the nuclear NIR luminosity permit us to derive the position of PGC 50427 on the known $\tau-M V$ diagram. The simultaneous observations for the broad-line region and dust thermal emission demonstrate that the innermost dust torus is located outside the BLR in PGC 50427, supporting the unified scheme for AGNs.
\end{abstract}

Key words. galaxies: active - galaxies: Seyfert - galaxies: distances and redshifts - galaxies: individual: PGC 50427

\section{Introduction}

The existence of a dusty structure with a torus-like geometry surrounding the broad-line region (BLR) and the central accreting super massive black hole (SMBH) play a fundamental role in the framework of a unified model for active Galactic nuclei (AGN; Antonucci 1993). Its presence would explain the observed differences in the spectra of type 1 and 2 Seyfert galaxies together with the strong emission observed at near-, mid-, and far-infrared wavelengths (Alonso-Herrero et al. 2001; Haas et al. 2003, 2004; Siebenmorgen et al. 2005).

Evidence for the surrounding hot dust torus rests indirectly on optical spectropolarimetric observations (e.g., Antonucci \& Miller 1985; Barvainis 1987; Kobayashi et al. 1993). A direct detection of the putative dust torus is more difficult since the internal structure of AGNs is spatially unresolved using single telescopes. Infrared long-baseline interferometric observations have been able to determine the size of the dust torus for a few nearby AGNs (e.g., Wittkowski et al. 2004; Tristram et al. 2007; Pott et al. 2010).

* Based on observations made with the Southern African Large Telescope (SALT).
The only method available for studying the origin and morphology of the BLR and the dust torus independent of the spatial resolution of the instrument is reverberation mapping (RM, Lyutyi \& Cherepashchuk 1972; Cherepashchuk \& Lyutyi 1973; Blandford \& McKee 1982; Gaskell \& Sparke 1986; Peterson 1993; Peterson et al. 2004). In this method one measures the light travel time between the accretion disk (AD) and the BLR and/or hot dust. The hot AD produces a variable continuum emission, and this variability is observed with a time delay $\left(\tau_{\mathrm{BLR}}=R_{\mathrm{BLR}} / c\right)$ in the studied broad emission lines of the BLR. Similarly, if the dust torus is located at some radial distance $R_{\text {dust }}$ around the hot AD it will reprocess the UV/optical radiation to thermal near-infrared (NIR) radiation with a characteristic time delay $\tau_{\text {dust }}=R_{\text {dust }} / c$. Reverberation mapping has revealed the size of the BLR, the black hole mass, and Eddington ratios in about 50 AGNs (see Du et al. 2014, and references therein).

The torus reacts to the $\mathrm{AD}$ variability in a wide range of time, from days to decades (e.g., Glass 2004; Suganuma et al. 2006; Pozo Nuñez et al. 2014a; Koshida et al. 2014). From dust reverberation measurements, which are principally based on the cross-correlation analysis between the optical $(V, 0.55 \mu \mathrm{m})$ and NIR $(K, 2.2 \mu \mathrm{m})$ light curves, a correlation between the innermost radius of the torus and the square root of the optical 
luminosity has been shown $\left(R_{\text {dust }} \propto L^{0.5}\right.$; Glass 2004; Minezaki et al. 2004; Suganuma et al. 2006; Koshida et al. 2014). The dust is heated by the accretion disk (AD) up to its maximum sublimation temperature $(\sim 1500 \mathrm{~K})$. The correlation and sublimation temperature are consistent with the theoretical prediction of the dust sublimation radius ( $R_{\text {sub }}$, Barvainis 1992), but is systematically smaller by a factor of three than the sublimation radius $R_{\text {sub }}$ predicted for graphite dust grains with a size of $0.05 \mu \mathrm{m}$ in radius (Oknyanskij et al. 1999; Kishimoto et al. 2007). Some modified dust geometries involve BLR associated dust due to winds or outflows from the accretion disk (Konigl \& Kartje 1994; Elitzur \& Shlosman 2006; Czerny \& Hryniewicz 2011).

Alternatively, theoretical simulations shows that an anisotropically illuminated dust torus, caused by an optically thick $\mathrm{AD}$, places the inner concave region of the torus closer to the outer edge of the AD. Thereby increasing the response time of the torus, and thus explaining the systematic difference of the time delay with respect to the torus radius measured from time delay and from the sublimation temperature under an isotropically assumption (Kawaguchi \& Mori 2010, 2011). In the case of the Seyfert 1 galaxy WPVS48, Pozo Nuñez et al. (2014a) argue that the sharp NIR echo observed is due to a geometrically and optically thick torus seen nearly face-on. In this scenario the observer only sees the facing rim of the torus wall, which lies closer to the observer than the torus equatorial plane and therefore leads to an observed foreshortened lag effect.

While great theoretical progress has been made, only a handful of observational measurements of the dust reverberation radius have been obtained over recent years. Moreover, as noted in Suganuma et al. (2006), the importance of simultaneous BLR and dust torus size measurements provide an important step forward to test and constrain the actual paradigm of unification for AGNs.

More recently, photometric reverberation mapping (PRM) has been revisited and used as an efficient alternative to determine the BLR size, black hole masses and host-subtracted AGN luminosities (Haas et al. 2011; Pozo Nuñez et al. 2012, 2013). Through the combination of broad- and narrowband data, this method is used to measure the time delay between the triggering continuum variations and the BLR emission line response, which has previously been isolated by the subtraction of the underlying continuum determined from the broadband filter data and/or through a single spectrum contemporaneous with the campaign.

Feature-rich PRM light curves allow us to infer the basic geometry of the BLR, whether it is spherical or disk-like, and can thus constrain the unknown geometrical factor needed in converting the time lag and velocity width into a black hole mass (Pozo Nuñez et al. 2014b). Furthermore, the use of broadband data alone has been tested with satisfactory results (Chelouche \& Daniel 2012; Chelouche et al. 2012; Edri et al. 2012; Pozo Nuñez et al. 2013; Chelouche \& Zucker 2013). In this method, two suitable chosen broadband filters are used to trace the continuum variations and to catch the emission line and continuum with the removal of the continuum performed in the crosscorrelation domain.

PGC 50427 has been classified as a Seyfert 1 galaxy and is located at a distance of $102 \mathrm{Mpc}$ (Véron-Cetty \& Véron 2010). In this paper we present the results of a multi-year monitoring campaign carried out on the nucleus of the Seyfert 1 galaxy PGC 50427. We use Photometric Reverberation Mapping in combination with dust-reverberation mapping to determine the black hole mass, the size of the BLR and dust torus for the first time in this source.

\section{Observations and data reduction}

The optical and near-infrared monitoring campaign of PGC 50427 were carried out using the VYSOS-6, BEST-II, BMT and IRIS telescopes (for more details see Sects. 2.1-2.2) located at the Universitätssternwarte Bochum observatory, near Cerro Armazones, the future location of the ESO Extreme Large Telescope (ELT) in Chile ${ }^{1}$.

In addition to the photometric observations, one single epoch spectrum was acquired using the Robert Stobie Spectrograph (RSS) at the Southern African Large Telescope (SALT). The timeline of all the observations in our campaign is shown in Fig. 1.

PGC 50427 lies at redshift $z=0.0236$, therefore the $\mathrm{H} \alpha$ emission line falls into the SII $6721 \pm 30 \AA$ and $6700 \pm 60 \AA$ narrowband (NB) filters. The characteristics of the source and the Galactic foreground extinction values are listed in Table 1. Figure 2 shows the position of the NB filters with respect to the $\mathrm{H} \alpha$ emission line together with the effective transmission of the other optical filters used.

\subsection{Optical monitoring}

\section{VYSOS-6}

Broadband Johnson $B$ (4330 $)$, Sloan-band $r(6230 \AA)$, and narrowband SII $(6721 \pm 30 \AA$, the position of the redshifted $\mathrm{H} \alpha$ line) observations were carried out during a monitoring campaign between February 18 and September 01, 2011, with the robotic VYSOS-6 telescope. The VYSOS-6 telescope consist of two $15 \mathrm{~cm}$ refractors (Takahashi TOA 150F OrthoApo-chromat Triplet) installed on a common equatorial mount (Bisque Paramount ME). Both refractors are equipped with an Apogee ALTA U16M $4096 \times 4096$ pixel CCD, providing a field of view $(\mathrm{FoV})$ of about $2.7^{\circ} \times 2.7^{\circ}$. More information about the telescope and the instrument has been published by Haas et al. (2012).

The data reduction was standardized, including bias, dark current, flatfield, astrometry and astrometric distortion corrections performed with IRAF $^{2}$ in combination with SCAMP (Bertin 2006) and SWARP (Bertin et al. 2002) routines. A 7".5 diameter aperture was used to extract the flux, which was normalized and converted into photometric fluxes through the comparison with 17 non-variable stars located on the same images within $30^{\prime}$ around the AGN and of similar brightness as the AGN. Absolute calibration was performed using standard reference stars from Landolt (2009) observed on the same nights as the AGN, considering the atmospheric (Patat et al. 2011) and Galactic foreground extinction (Schlafly \& Finkbeiner 2011) corrections. The reader is referred to Pozo Nuñez et al. (2013) for further information about reduction and light curve extraction process.

\section{BEST-II}

Broadband $B(4330 \AA)$ and $R(7000 \AA)$ Johnson observations were carried out during a monitoring campaign between

\footnotetext{
1 http://www . astro.ruhr-uni-bochum.de/astro/oca/

2 IRAF is distributed by the National Optical Astronomy Observatory, which is operated by the Association of Universities for Research in Astronomy (AURA) under cooperative agreement with the National Science Foundation.
} 


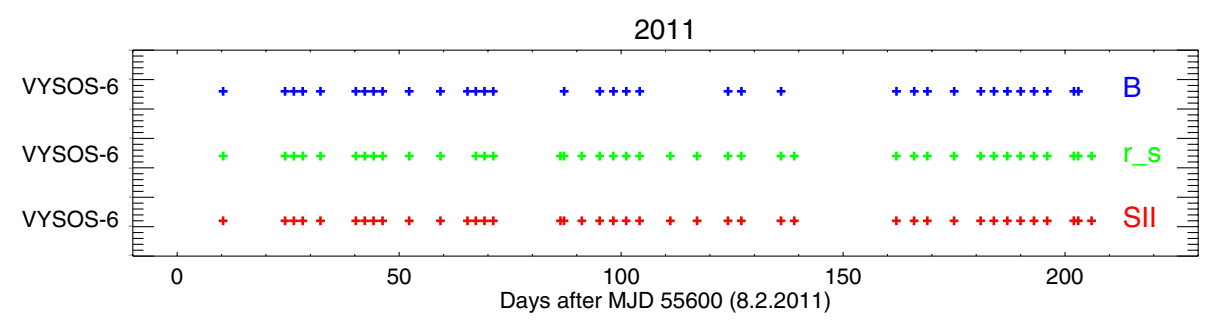

2013

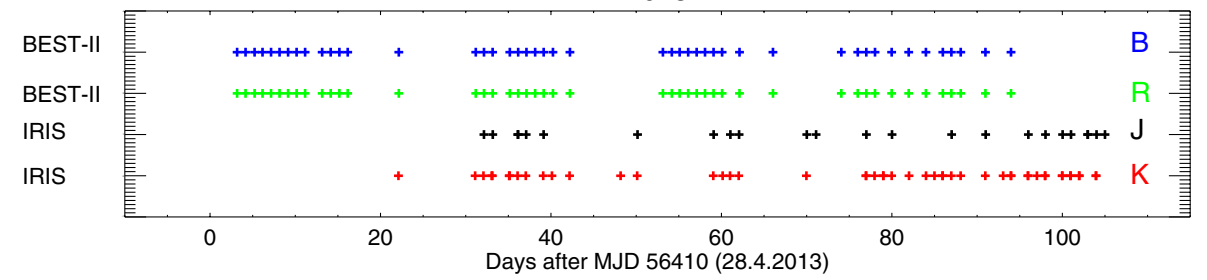

2014

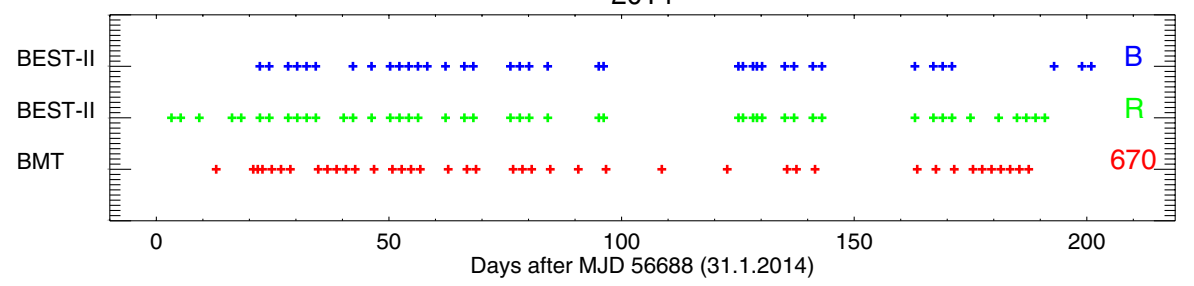

Fig. 1. Timeline of our campaign on PGC 50427. The days in which the telescopes made observations are indicated by crosses.

Table 1. Characteristics of PGC 50427.

\begin{tabular}{cccccccccc}
\hline \hline$\alpha(2000)^{a}$ & $\delta(2000)^{a}$ & $z^{a}$ & $\begin{array}{c}D_{L}{ }^{a} \\
(\mathrm{Mpc})\end{array}$ & $\begin{array}{c}B-V^{b} \\
(\mathrm{mag})\end{array}$ & $\begin{array}{c}M_{\mathrm{abs}}{ }^{b} \\
(\mathrm{mag})\end{array}$ & $\begin{array}{c}A_{u}{ }^{c} \\
(\mathrm{mag})\end{array}$ & $\begin{array}{c}A_{B}{ }^{c} \\
(\mathrm{mag})\end{array}$ & $\begin{array}{c}A_{R}{ }^{c} \\
(\mathrm{mag})\end{array}$ & $\begin{array}{c}A_{r}{ }^{c} \\
(\mathrm{mag})\end{array}$ \\
\hline $14: 08: 06.7$ & $-30: 23: 53.7$ & 0.024 & 102.0 & 0.40 & -20.6 & 0.252 & 0.215 & 0.129 & 0.135 \\
\hline
\end{tabular}

Notes. ${ }^{(a)}$ Values from NED database; ${ }^{(b)}$ Véron-Cetty \& Véron $(2010) ;{ }^{(c)}$ Schlafly \& Finkbeiner (2011).

May 01 and July 30, 2013, and between February 03 and August 19, 2014, with the robotic $25 \mathrm{~cm}$ Berlin Exoplanet Search Telescope-II (BEST-II). The BEST-II telescope is equipped with a Peltier-cooled $4096 \times 4096$ pixel Finger Lakes Imager CCD KAF-16801, yielding a field of view of $1.7^{\circ} \times 1.7^{\circ}$ with a pixel size of $9 \mu \mathrm{m}$. More information about the telescope and the instrument has been published by Kabath et al. (2009). The data were reduced and calibrated following the same procedures as for the VYSOS-6 telescope.

\section{BMT}

Narrowband $(6700 \pm 120 \AA$, hereafter denoted as 670) Ashahi observations were carried out simultaneously to the broadband monitoring campaign in 2014, using the robotic $40 \mathrm{~cm}$ Bochum Monitoring Telescope (BMT). The BMT telescope is equipped with a two-stage thermoelectric cooled $3072 \times 2038$ pixel CCD SBIG STL-6303, yielding a field-of-view of $41^{\prime} \times 27^{\prime}$ with a pixel size of $9 \mu \mathrm{m}$. The data were reduced and calibrated following the same procedures as in the broadband analysis. More information about the telescope and the instrument has been published by Ramolla et al. (2013).

\section{SALT}

The optical spectrum of PGC 50427 was observed using the $11 \mathrm{~m}$ Southern African Large Telescope (SALT) on May 10, 2013
(Proposal Code: 2013-1-HETGU-001). The observations were performed with the Robert Stobie Spectrograph (RSS) mounted at the prime focus of the SALT. We used the PG0900 grating with a resolving power of 1065 at $6050 \AA$, and the spectrum covers a wavelength range between $3200-9000 \AA$. The spectrum was taken in two identical consecutive exposures of $10 \mathrm{~min}$ each through the $2^{\prime \prime} \times 8^{\prime}$ longslit PL 0200N001 at a parallactic angle. The detector was operated in normal readout mode with a $2 \times 2$ binning. A Xe spectrum was obtained after the object exposure for wavelength calibration. For flux calibration we observed the standard star G24-9. After the bias subtracted file provided by the SALT pipeline, we used standard IRAF routines for flat-field correction, cosmic ray rejection, 2D-wavelength calibration, night sky subtraction and flux calibration. For the 1D spectrum, we combined 7 columns, corresponding to $1.7738^{\prime \prime}$ $\left(0.1267^{\prime \prime}\right.$ per unbinned pixel). The reduced spectrum is shown in Fig. 2.

\subsection{Near-infrared monitoring}

\section{IRIS}

Near-infrared (NIR) $J(1.25 \mu \mathrm{m})$ and $K \mathrm{~s}(2.15 \mu \mathrm{m}$, hereafter denoted as $K$ ) observations were carried out between May 20 and August 11, 2013 using the $0.8 \mathrm{~m}$ Infrared Imaging System (IRIS) telescope. IRIS is equipped with a HAWAII-1 nitrogen-cooled 


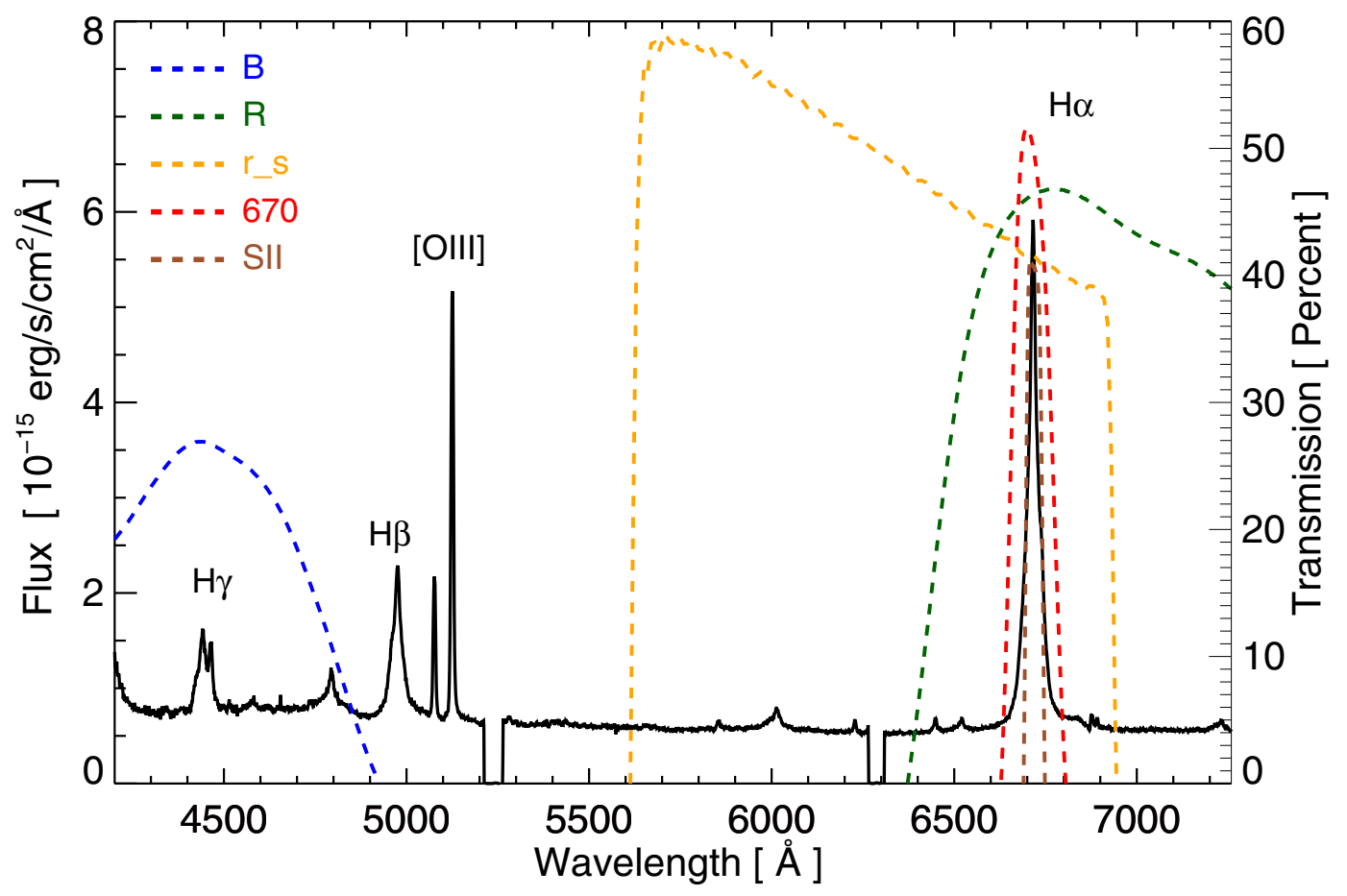

Fig. 2. SALT spectrum of PGC 50427. For illustration, the band passes of the filters used for the photometric monitoring are shown (blue $B$-band, green $R$-band, orange $r_{\mathrm{s}}$-band, red 670-band, and brown for SII-band). The NB 670 and SII catches the redshifted H $\alpha$ line, its flux is composed by the contribution of about $85 \% \mathrm{H} \alpha$ line and $15 \%$ continuum. We note that for actual flux calculations the filter curves are convolved with the quantum efficiency of the Alta U16 and SBIG STL CCDs cameras.

detector array with $1024 \times 1024$ pixels, yielding a field of view of $12.5^{\prime} \times 12.5^{\prime}$ and a resolution of $0.74^{\prime \prime}$. $/$ pixel (Hodapp et al. 2010). Images were obtained by combining double cycles of $20 \mathrm{~s}$ exposure time acquired with the observing sequence object-skyobject. The images were reduced using IRAF routines. Because the sky background emission contribution is one of the most difficult step in NIR data reduction, the sky frames observed close to the AGN were subtracted from each science frame before flatfield and further corrections. One final image, resulting from the combination of all individual frames, is obtained in order to remove cosmic rays, hot pixeles and negative residuals from the sky-subtracted science frames. The data reduction steps after the sky background subtraction and correction for cosmic rays, hot pixels and negative residuals, are the same as outlined for the VYSOS-6 telescope, including astrometry and astrometric distortion correction with SCAMP and SWARP. Light curves were calculated relative to six non-variable stars located on the same field having similar brightness as the AGN. Photometric calibration was achieved by using 4 high-quality flag (AAA) Two Micron All Sky Survey (2MASS) stars appearing in the same field as the AGN. As already noted for the optical treatment of the data (Pozo Nuñez et al. 2013), analysis for different aperture photometry was performed considering the proper minimization of the host-galaxy contribution and a 7'.0 diameter aperture was chosen for further analysis.

\section{Results and discussion}

\subsection{Optical light curves and BLR geometry}

The optical light curves for campaigns 2011 and 2014 are shown in Fig. 3. The light curves are published at the CDS. In the campaign of 2011, the $B$-band shows a gradual flux increase of $20 \%$ from the beginning of March until a maximum is reached at the beginning of April (Fig. 3, left). After this overall maximum, the fluxes undergo an abrupt drop by about $40 \%$ until the end of May. Between the beginning of June and the end of August 2011, the fluxes are steady. The $r$-band light curve follow the same features than the $B$-band light curve albeit with a smaller variability amplitude. This is expected because of the larger constant hostgalaxy contribution. Moreover, the $r$-band also contains a contribution from the strong $\mathrm{H} \alpha$ emission line (Fig. 2). The narrow SII-band light curve follows the continuum dominated broadband light curves qualitatively, but we can see a 20 day delay compared to the continuum variability at the minimum observed at the end of June.

As already discussed in previous PRM studies, the narrowband contains, in addition to the $\mathrm{H} \alpha$ line, a contribution of the varying AGN continuum, which must be removed before applying cross-correlation techniques (Haas et al. 2011; Pozo Nuñez et al. 2012, 2013). In order to determine this contribution, we used the SII and $r$-band fluxes, previously calibrated to mJy, as is shown with the flux-flux diagram in Fig. 4. The $\mathrm{H} \alpha$ line is contributing, on average, about $70 \%$ of the total flux enclose in the SII-band, while the continuum contribution ( $r$-band) is about $30 \%$. Following the usual practice of narrowband PRM, we construct a synthetic $\mathrm{H} \alpha$ light curve by subtracting a fraction of the $r$-band light curve ( $\mathrm{H} \alpha=\mathrm{SII}-0.3 r)$, as illustred in Fig. 6. The $\mathrm{H} \alpha$ light curve was used afterwards to estimate the time delay. For this purpose, we used the discrete correlation function (DCF, Edelson \& Krolik 1988) to cross-correlate the continuum and the synthetic $\mathrm{H} \alpha$ emission line, taking into account possible bin size dependency ${ }^{3}$ (Rodriguez-Pascual et al. 1989).

\footnotetext{
3 The choice of a lower or higher time-bin size does not change the results if a well sampled data is used (Pozo Nuñez et al. 2012).
} 

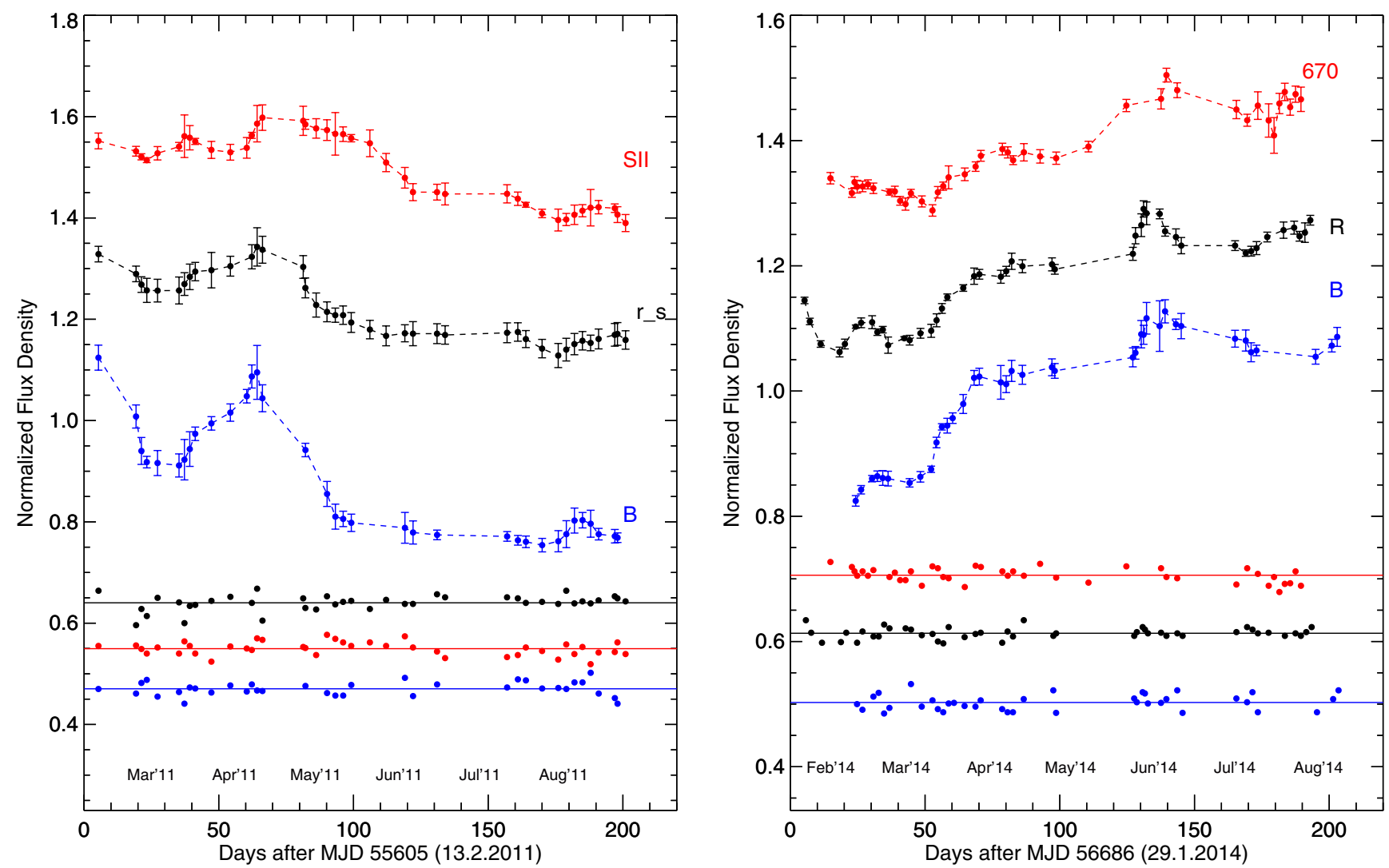

Fig. 3. Observed light curves of PGC 50427, as well as for some of the reference stars in the field of view for the period between February 2011 and September 2011 (left) and for the period between February 2014 and August 2014 (right). The light curves are vertically shifted for clarity.

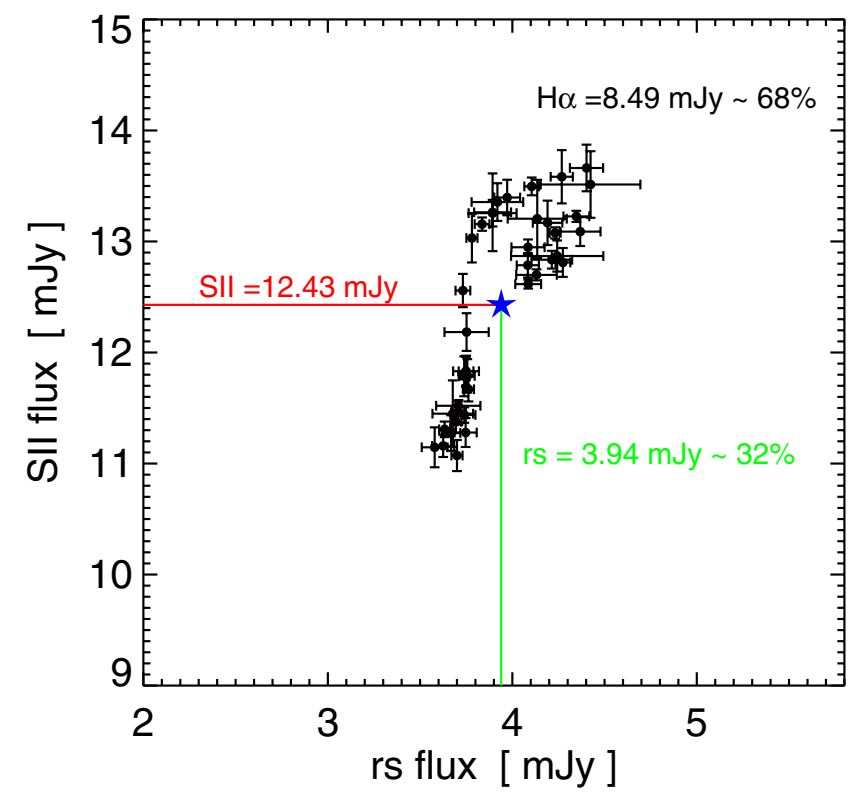

Fig. 4. Flux-flux diagram for the SII and $r$ filter. Black dots denote the measurement pair of each night during campaign 2011. The red and green lines represent the average flux in the SII and $r$ band respectively. Fluxes were measured using circular $7.5^{\prime \prime}$ apertures. The data are as observed and not corrected for extinction.

The centroid of the cross-correlation between the $B$-band and $\mathrm{H} \alpha$ light curves shows a time delay of 20.5 days (Fig. 8). Uncertainties in the time delay were calculated using the flux randomization and random subset selection method (FR/RSS,

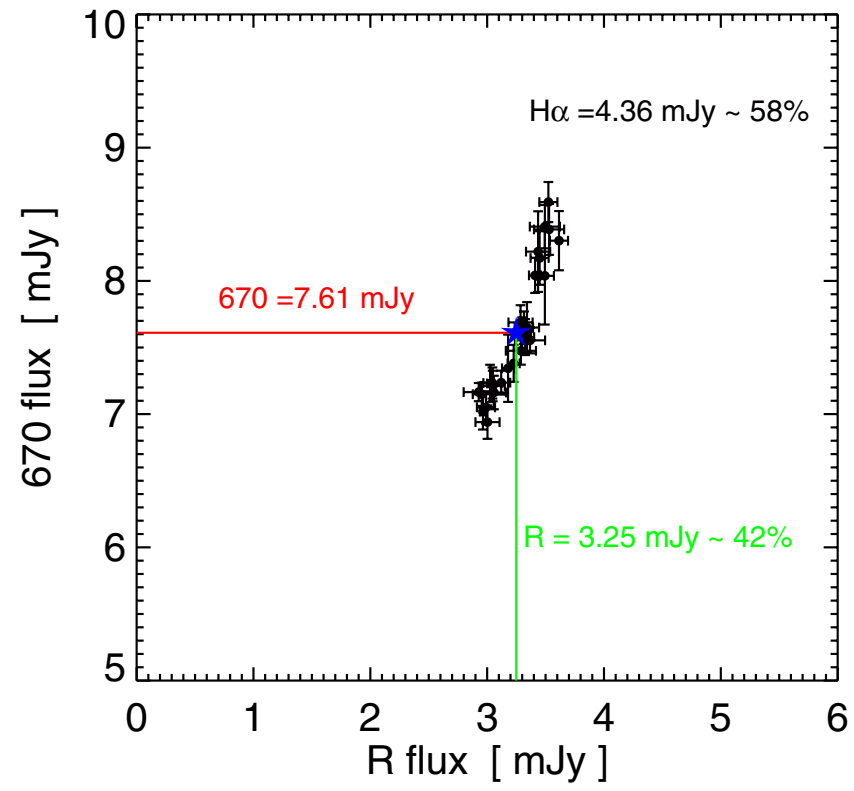

Fig. 5. Same as Fig. 4, but for 670 and $R$ data obtained during campaign 2014.

Peterson et al. 2004). From the observed light curves we create 2000 randomly selected subset light curves, each containing $63 \%$ of the original data points (the other fraction of points are unselected according to Poisson probability). The flux value of each data point was randomly altered consistent with its (normal-distributed) measurement error. We calculated the DCF for the 2000 pairs of subset light curves and the corresponding 


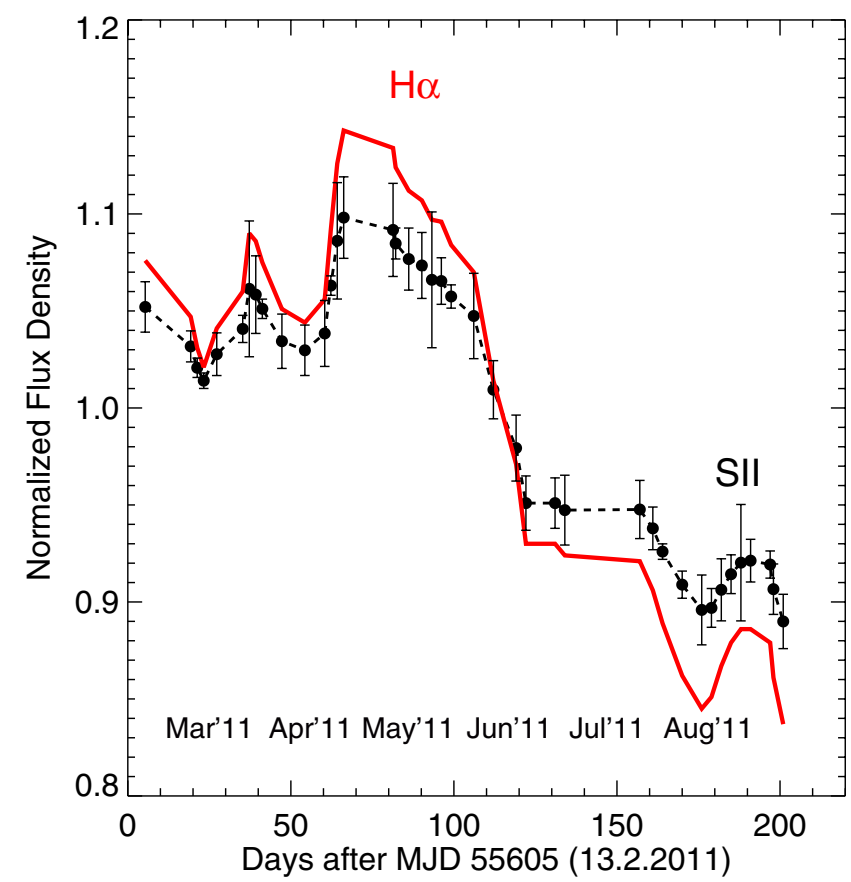

Fig. 6. $\mathrm{H} \alpha$ light curve (red) obtained after the continuum subtraction process performed on the SII light curve (black) during campaign 2011. The continuum fraction was obtained from the flux-flux diagnostic on the SII and $r$ bands; see text for details.

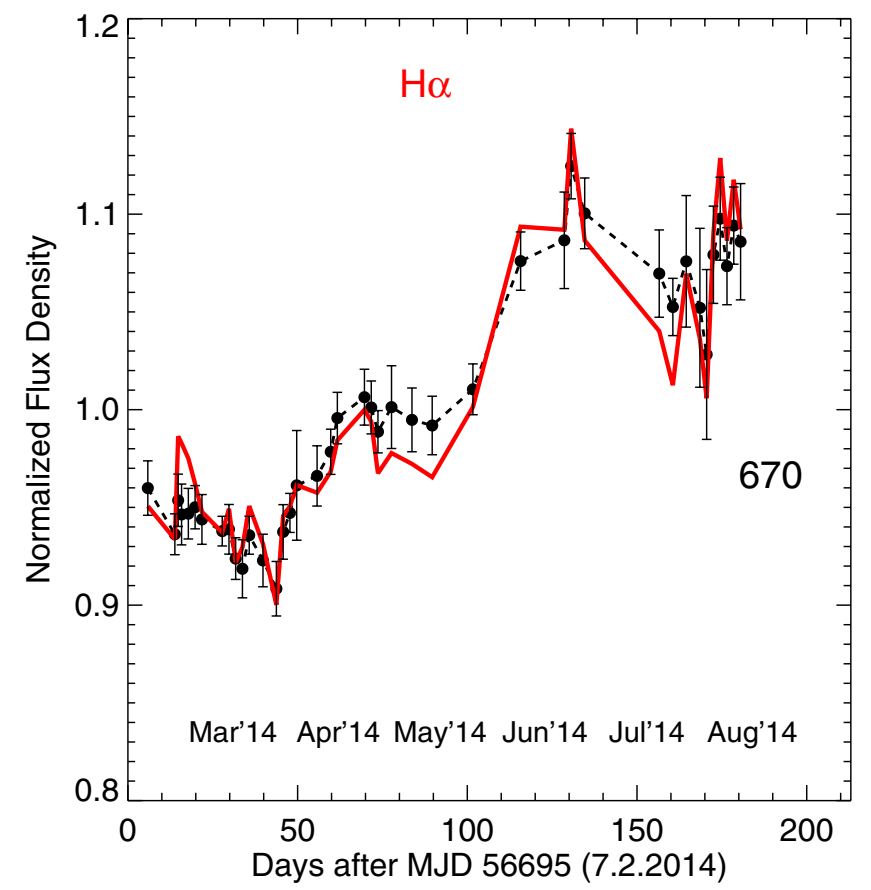

Fig. 7. Same as Fig. 6, but for 670 and $R$ data obtained during campaign 2014.

centroid. From this cross-correlation error analysis, we measure a median lag of $\tau_{\text {cent }}=20.4_{-1.0}^{+0.4} \mathrm{~B} / \mathrm{H} \alpha$. Correcting for the time dilation factor $(1+z=1.0236)$ we obtain a rest frame lag of $19.9 \pm 0.68$ days $B / \mathrm{H} \alpha$.

In the campaign of 2014 , we see a steep $B$-band rise at the beginning of April by about $20 \%$ which is also observed in the $R$ band, but with a smaller amplitude (Fig. 3, right). In contrast to the steep $B$-band flux increase, the narrow 670 -band rise appears

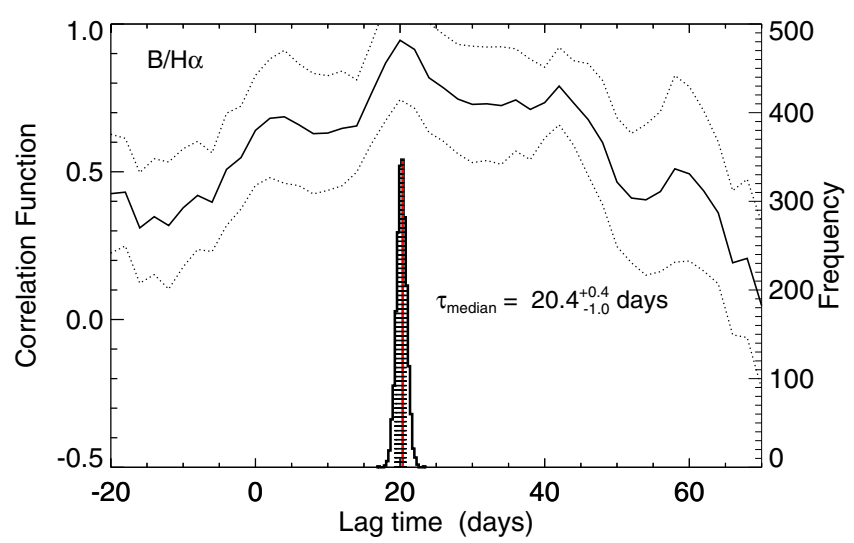

Fig. 8. Cross correlation of $B$ and $\mathrm{H} \alpha$ light curves for campaign 2011. The dotted lines indicate the error range $( \pm 1 \sigma)$ around the cross correlation. The centroid was calculated above the correlation level at $r \geq 0.8 r_{\max }$. The histogram shows the distribution of the centroid lag obtained by cross correlating 2000 flux randomized and randomly selected subset light curves (FR/RSS method). The black shaded area marks the $68 \%$ confidence range used to calculate the errors of the centroid.

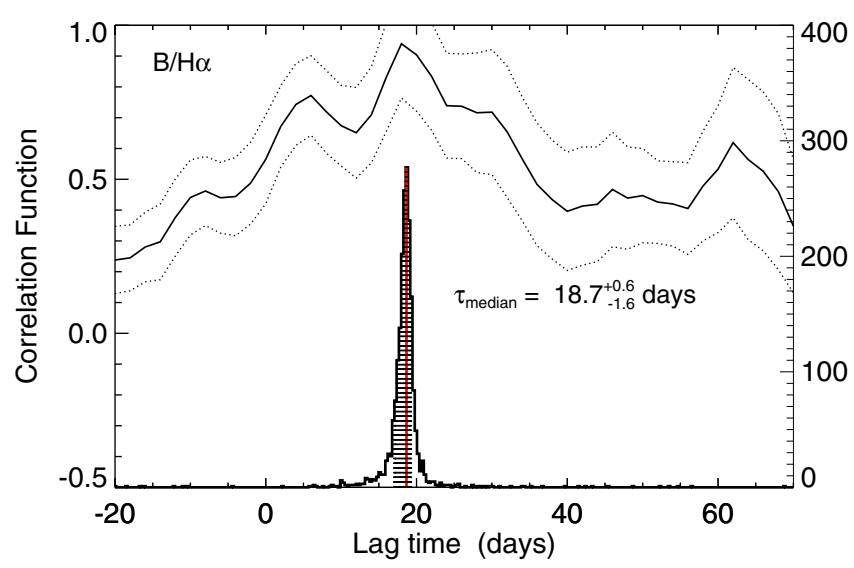

Fig. 9. Same as Fig. 8, but for campaign 2014.

stretched until the end of April with an amplitude of about $13 \%$, suggesting a time delay of the $\mathrm{H} \alpha$ line of 15-20 days. After a period of constant flux, the $B$ and $R$ light curves show a small sharp bump in June of about $10 \%$, which is observed about 15 days later in the 670-band. However, because of insufficient data obtained at this period in the 670-band, the delay cannot be measured as precisely as for the first. We have isolated the $\mathrm{H} \alpha$ emission line by the subtraction of the underlying continuum following a similar procedure as for the 2011 campaign. The $\mathrm{H} \alpha$ line is contributing, on average, about $60 \%$ of the total flux enclosed in the 670-band, while the continuum contribution ( $R$-band) is about $40 \%$ (Fig. 5). We construct a synthetic $\mathrm{H} \alpha$ light curve by subtracting a fraction of the $R$-band light curve ( $\mathrm{H} \alpha=670-0.4 R$ ), as illustred in Fig. 7. The centroid from the cross-correlation between the $B$-band and $\mathrm{H} \alpha$ shows a time delay of 19.3 days (Fig. 9). From the FR/RSS cross-correlation error analysis, we measure a median lag of $\tau_{\text {cent }}=18.7_{-1.5}^{+0.6}$. Correcting for the time dilation factor we obtain a rest frame lag of $18.3 \pm 1.03$ days. The deduced values for the time delay obtained at two different epochs are in qualitative agreement, and consistent with the small luminosity variations in the AGN $(\sim 10 \%)$ accross the monitoring campaign (see Sect. 3.3). We note that the relatively prominent contribution of the $\mathrm{H} \gamma$ emission line (Fig. 2 ) to the $B$-band $(\sim 10 \%)$ may result in a slightly 


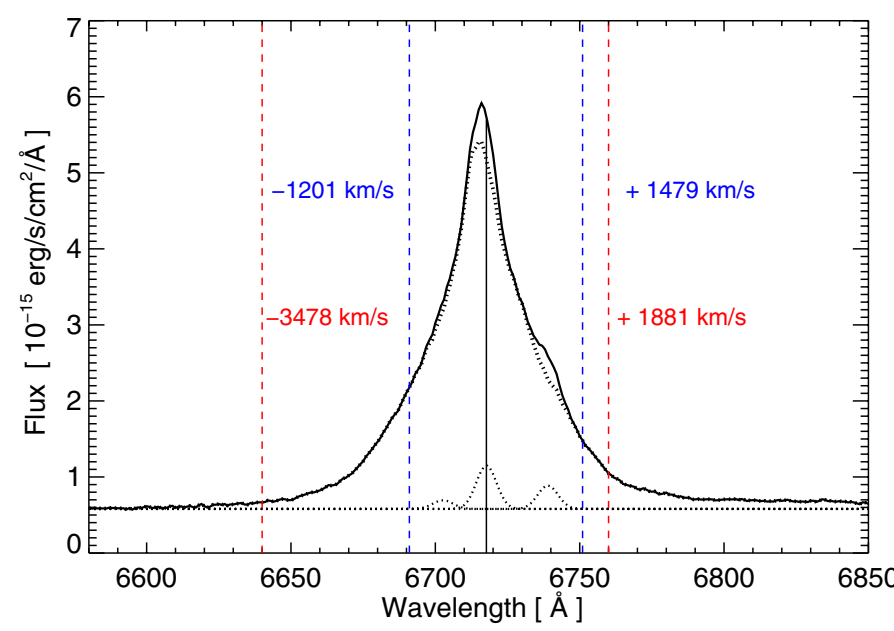

Fig. 10. SALT spectrum of PGC 50427, zoomed onto the $\mathrm{H} \alpha$ line. The dotted black curve represents the spectrum after subtracting the narrow [NII] $\lambda \lambda 6548,6583$ emission lines. The narrow $\mathrm{H} \alpha$ and [NII] models are shown in dotted lines at the bottom of the $\mathrm{H} \alpha$ profile. The NB 670 and SII filters efectively covers the line between velocities $-3478 \mathrm{~km} \mathrm{~s}^{-1}$ and $+1881 \mathrm{~km} \mathrm{~s}^{-1}$ (red dotted lines) and $-1201 \mathrm{~km} \mathrm{~s}^{-1}$ and $+1479 \mathrm{~km} \mathrm{~s}^{-1}$ (blue dotted lines) respectively.

higher time lag centroid from the DCF as the $B$-band light curve contains not only the AGN continuum, but also the lagged $\mathrm{H} \gamma$ signal. In the net effect, our estimate $B / \mathrm{H} \alpha$ may underestimate the true $B / \mathrm{H} \alpha$ time delay. However, the current data does not allow us to quantify this effect; this would requiere the use of emission line free continuum bands, for instance with the ultraviolet band.

\subsection{Central black hole mass}

Assuming that the BLR emiting gas clouds are in virialized motion around the central black hole, the mass of the black hole can be estimated as $M_{\mathrm{BH}}=f \cdot R_{\mathrm{BLR}} \cdot \sigma_{V}^{2} / G$. The velocity $\sigma_{V}$ of the emission-line region is determined from the line dispersion $\left(\sigma_{\text {line }}\right)$ or from the full width at half maximum (FWHM) of the line profile. The scaling factor $f$ depends on the geometry and kinematics of the BLR. Most of the results presented in previous reverberation studies have been carried out considering only the virial product $c \tau \sigma_{V}^{2} / G$, i.e., assuming a scaling factor $f=1$ (Peterson et al. 2004, and references therein).

The broad $\mathrm{H} \alpha$ emission line is blended with the narrow $\mathrm{H} \alpha$ and $[\mathrm{NII}] \lambda \lambda 6548,6583$ narrow emission lines. In order to remove the narrow components, we model the [SII] $\lambda \lambda 6716,6731$ doublet with a multi-Gaussian profile as described in Greene $\&$ Ho (2004). The model is shifted and scaled to fit the $\mathrm{H} \alpha+$ [NII] $\lambda \lambda 6548,6583$ narrow lines and subtracted from the observed broad $\mathrm{H} \alpha$ line profile as shown in Fig. 10. The ratio of the [NII] lines is fixed at the theoretical value of 2.96 and the relative positions of the narrow $\mathrm{H} \alpha$ and [NII] lines are determined by their laboratory wavelenghts. After removing the narrow emission lines, the $\mathrm{H} \alpha$ profile was isolated by the subtraction of a linear continuum fit, obtained through interpolation between two continuum segments on either end of the line. Figure 10 illustrate the original $\mathrm{H} \alpha$ emission line profile together with the subtracted narrow emission line profiles. The velocity dispersion after removal of narrow lines is $\sigma_{\text {line }}=1020 \mathrm{~km} \mathrm{~s}^{-1}$, which has been corrected for instrumental velocity dispersion to obtain an intrisic profile width. The feasibility of the use of single-epoch (SE) spectra for the black hole mass determination has been

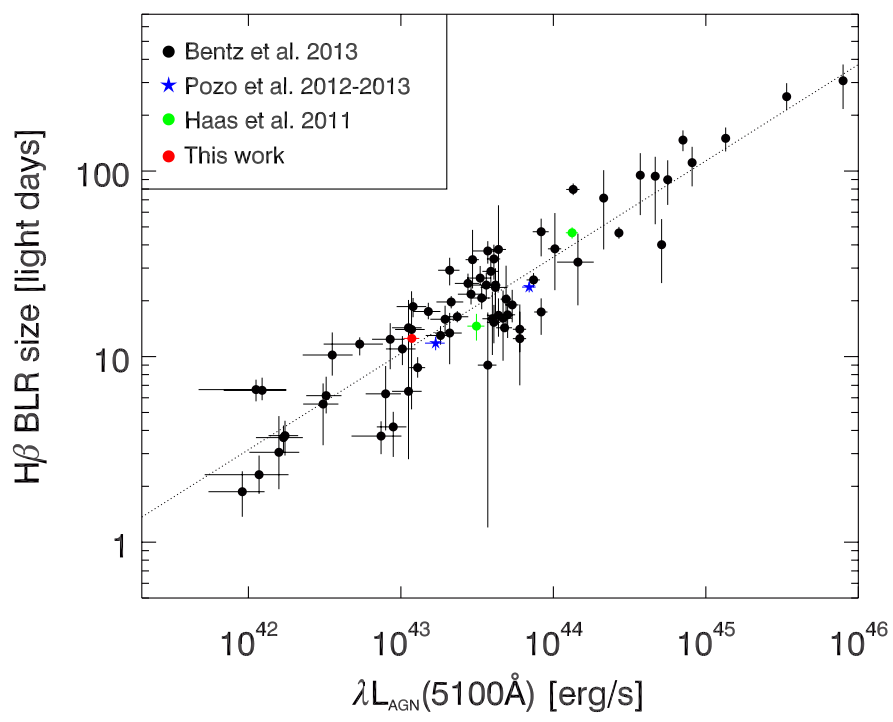

Fig. 11. $R_{\mathrm{BLR}}-L$ relationship from data of Bentz et al. (2013) (black dots) with a fitted slope $\alpha=0.533$ (dotted line). The diagram also contain the objects from previous photometric reverberation mapping campaigns (Haas et al. 2011, green dots; Pozo Nuñez et al. 2012, 2013, blue stars, and this article, red filled circle).

established in previous investigations. On average, uncertainties of $\sim 30 \%$ have been reported for black hole mass determination from single epoch spectra measurements (e.g. Vestergaard 2002; Woo et al. 2007; Denney et al. 2009).

Using the derived time delay $\tau=18.3 \mathrm{~d}$ for epoch 2014 and the velocity dispersion (with $30 \%$ uncertainty), the virial black hole mass is $M_{\text {virial }}=(3 \pm 2) \times 10^{6} M_{\odot}$. Considering the factor $f=5.5 \pm 1.8$, based on the asumption that AGNs follow the same $M_{\mathrm{BH}}-\sigma_{*}$ relationship (Onken et al. 2004), we determine a central black hole mass $M_{\mathrm{BH}}=(17 \pm 11) \times 10^{6} M_{\odot}$.

Assuming a symmetric BLR, the dimensionless factor $f$ depends on the unknown inclination of the BLR $\left(f=\frac{2 \cdot \ln 2}{\sin ^{2} i}\right)$. The optical and the $\mathrm{H} \alpha$ emission line variability observed in PGC 50427 suggest a disk-like BLR geometry with low inclination $i \leq 30^{\circ}$ (Pozo Nunez et al., in prep.), hence the geometryscaling factor $f$ may be much higher than the commonly used $f=5.5$. Therefore the black hole mass derived here should be considered as lower limit.

\subsection{Host-subtracted nuclear luminosity and the BLR size- luminosity relationship}

To determine the AGN luminosity free of host galaxy contributions, we applied the flux variation gradient (FVG) method, originally proposed by Choloniewski (1981) and later modified by Winkler et al. (1992). A detailed description of the FVG method on PRM data is presented in Pozo Nuñez et al. (2012). In this method the fluxes obtained through different filters and same apertures are plotted in a flux-flux diagram. The fluxes follow a linear slope representing the AGN color, while the slope of the nuclear host galaxy contribution (including the contribution from the narrow line region (NLR)) lies in a well defined range $\left(0.4<\Gamma_{B V}^{\text {host }}<0.53\right.$, for 8 .' 3 aperture and redshift $z<0.03$, Sakata et al. 2010). The AGN slope is determined through a linear regression analysis. Averaging over the intersection area between the AGN and the host galaxy slopes yields the actual host galaxy contribution at the time of the monitoring campaign. Figure 12 shows the FVG diagram for the $B$ and 

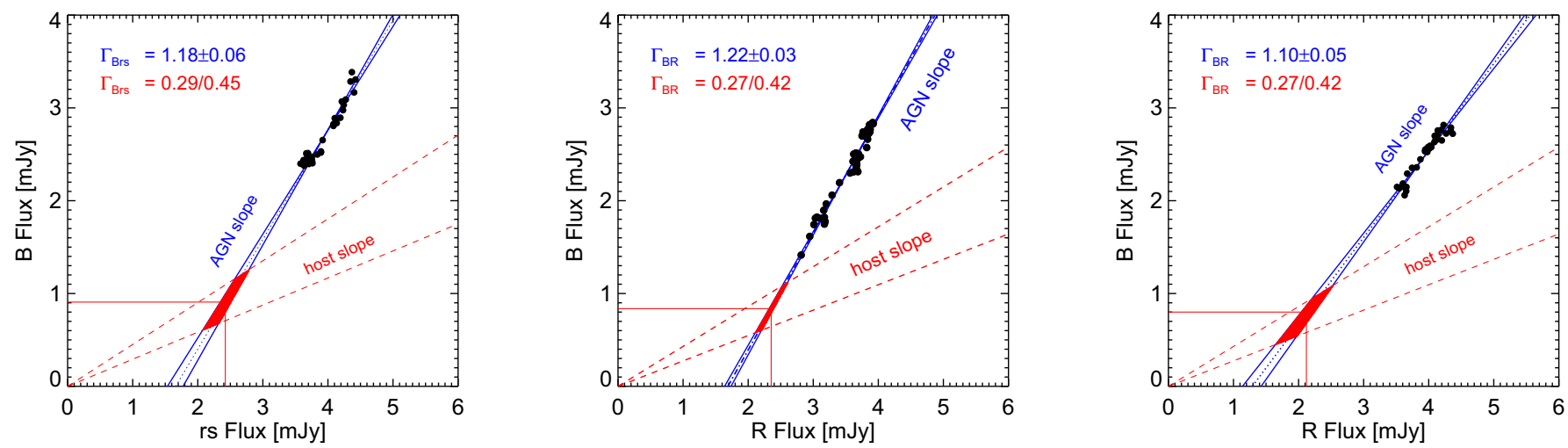

Fig. 12. Flux variation gradient diagrams for 2011 (left), 2013 (middle) and 2014 (right) campaigns. The solid lines delineate the bisector regression model yielding the range of the AGN slope. The dashed lines indicate the range of host slopes determined by Sakata et al. (2010) for 11 nearby AGN. The intersection between the host galaxy and AGN slope (red area) gives the host galaxy flux at the time of the campaign in both bands.

$r$ fluxes corresponding to campaign 2011 and for the $B$ and $R$ fluxes corresponding to campaign 2013/2014 obtained during the same nights and through a 7".5 aperture. All the fluxes has been corrected for Galactic foreground extinction.

The bisector linear regression method yields a linear gradient of $\Gamma_{B R_{\mathrm{s}}}=1.18 \pm 0.06, \Gamma_{B R}=1.22 \pm 0.03$ and $\Gamma_{B R}=1.10 \pm 0.05$ for campaigns in 2011, 2013, and 2014, respectively. The results are consistent, within the uncertainties, with the gradients obtained for other Seyfert 1 galaxies by Winkler et al. (1992) and Sakata et al. (2010). The AGN fluxes at the time of the monitoring can be determined by subtracting the host galaxy fluxes from the total fluxes. The host galaxy subtracted average AGN fluxes and the host galaxy flux contribution of PGC 50427 are listed in Table 2. Also listed in Table 2 are the interpolated rest frame $5100 \AA$ fluxes and the monochromatic AGN luminosity $\lambda L_{\lambda(\mathrm{AGN})}$ at $5100 \AA$ obtained at the distance of $102 \mathrm{Mpc}$. The rest frame flux at $5100 \AA$ was interpolated from the host-subtracted AGN fluxes in both bands, assuming for the interpolation that the AGN spectral energy distribution (SED) is a power law $\left(F_{v} \propto v^{\alpha}\right)$ with a spectral index $\alpha=\log \left(f x_{\mathrm{AGN}} / f y_{\mathrm{AGN}}\right) / \log \left(v_{x} / v_{y}\right)$, where $v_{x}$ and $v_{y}$ are the effective frequencies in the $\mathrm{X}$ and $\mathrm{Y}$ bands, respectively. The error was determined by interpolation between the ranges of the AGN fluxes $\pm \sigma$ in both filters, respectively.

The position of PGC 50427 on the BLR size-luminosity diagram is shown in Fig. 11. The values of other galaxies are taken from Bentz et al. (2013) and from previous photometric reverberation mapping campaigns (Haas et al. 2011; Pozo Nuñez et al. 2012, 2013). For this figure we converted the measured $\mathrm{H} \alpha$ size into the size of the $\mathrm{H} \beta$ BLR using the weighted mean ratio for the time lag $\tau(H \alpha): \tau(H \beta): 1.54: 1.00$, obtained by Bentz et al. (2010) from the Lick AGN Monitoring Program of 11 low-luminosity AGN.

\subsection{Infrared light curves and dust-torus size}

Figure 13 depicts the optical and near-infrared normalized light curves of the nucleus of PGC 50427 obtained during the 2013 campaign. The light curves are published at the CDS. To deconvolve the host galaxy and the nuclear flux contributions, we used the FVG method in the same way as described in Pozo Nuñez et al. (2014a). The SED of the variable component remain constant with time, and in consequence the slopes obtained from the optical and NIR flux ratios $(B / R$ and $J / K)$ allow us to separate the AGN flux through the use of a well-defined range of host galaxy colors (Glass 2004; Sakata et al. 2010). Figure 14

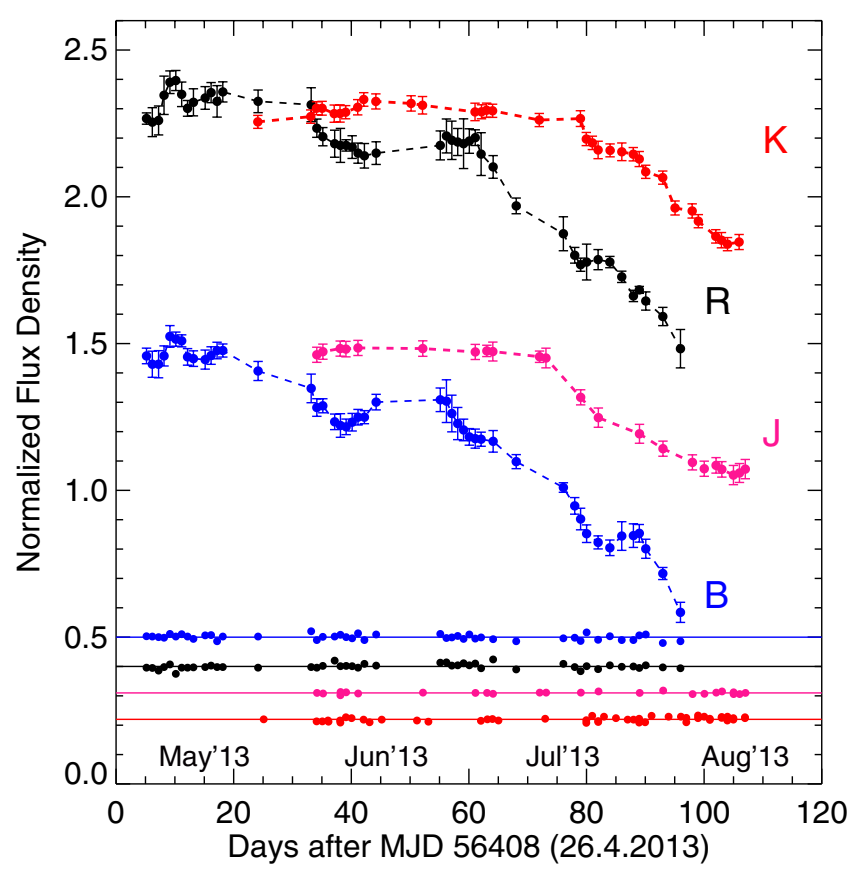

Fig. 13. Optical and NIR host galaxy subtracted light curves obtained between May and August 2013.

shows the $J$ flux plotted against the $K$ flux for each night. The bisector linear regresion method yields a linear gradient of $\Gamma_{J K}=0.38 \pm 0.05$ which correspond to a nuclear color $J-K=1.97 \pm 0.07$, and is consistent with the FVG colors of Seyfert galaxies $(J-K \sim 2.3)$ measured by Glass (2004). Figure 15 shows the distribution of $J-K$ colors of Seyfert galaxies previously studied by Glass (2004) and the value for PGC 50427 obtained in this work. The color of PGC 50427 correspond to a blackbody temperature of $1850 \mathrm{~K}$, which is in agreement with the sublimation temperature of hot pure graphite grains (Mor \& Netzer 2012), and supports the previous conclusions that IR emission observed from Seyfert galaxies is dominated by thermal radiation from the hot dust close to the central $\mathrm{AD}$ (Suganuma et al. 2006).

The optical $B$ and $R$-bands, which are mostly dominated by the AGN continuum ${ }^{4}$, show a similar varibility behavior. The

\footnotetext{
4 The $R$-band also contains a small contribution from the strong $\mathrm{H} \alpha$ emission line.
} 
Table 2. Total, host galaxy and AGN optical fluxes given in mJy for the different campaigns.

\begin{tabular}{ccccccc}
\hline \hline Campaign & Filter & $\begin{array}{c}\text { Total } \\
(\mathrm{mJy})\end{array}$ & $\begin{array}{c}\text { Host } \\
(\mathrm{mJy})\end{array}$ & $\begin{array}{c}\mathrm{AGN}^{a} \\
(\mathrm{mJy})\end{array}$ & $\begin{array}{c}f_{\mathrm{AGN}}((1+z) 5100 \AA \\
(\mathrm{mJy})\end{array}$ & $\begin{array}{c}\lambda L_{\lambda, \mathrm{AGN}} 5100 \AA \\
\left(10^{43} \mathrm{erg} \mathrm{s}^{-1}\right)\end{array}$ \\
\hline 2011 & $B$ & $2.71 \pm 0.12$ & $0.91 \pm 0.17$ & $1.80 \pm 0.20$ & $1.66 \pm 0.20$ & $1.18 \pm 0.14$ \\
& $r s$ & $3.95 \pm 0.11$ & $2.42 \pm 0.15$ & $1.53 \pm 0.19$ & & \\
2013 & $B$ & $2.36 \pm 0.10$ & $0.83 \pm 0.15$ & $1.53 \pm 0.18$ & $1.40 \pm 0.17$ & $1.00 \pm 0.12$ \\
& $R$ & $3.56 \pm 0.11$ & $2.35 \pm 0.12$ & $1.21 \pm 0.16$ & & \\
2014 & $B$ & $2.48 \pm 0.10$ & $0.80 \pm 0.13$ & $1.68 \pm 0.16$ & $1.72 \pm 0.17$ & $1.22 \pm 0.12$ \\
& $R$ & $3.94 \pm 0.12$ & $2.12 \pm 0.15$ & $1.82 \pm 0.19$ & & \\
\hline
\end{tabular}

Notes. ${ }^{(a)}$ AGN fluxes values $f_{\text {AGN }}=f_{\text {total }}-f_{\text {host }}$ with uncertainty range $\sigma_{\text {AGN }}=\left(\sigma_{\text {total }}^{2}+\sigma_{\text {host }}^{2}\right)^{0.5}$.

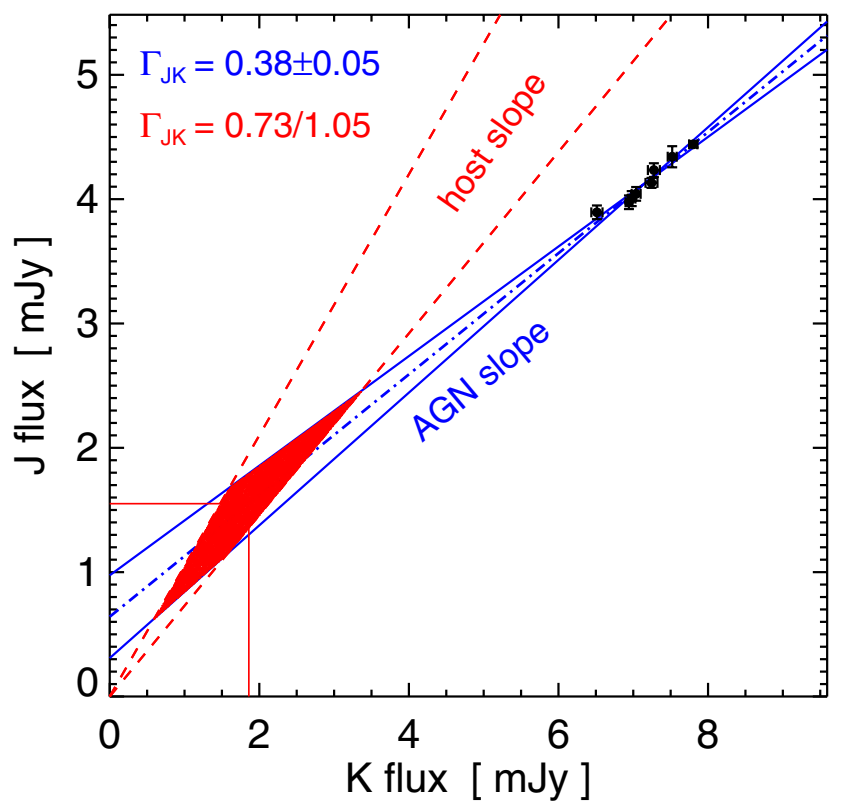

Fig. 14. Flux variation gradient diagram in the NIR. The data are represented by the black dots. The solid blue lines represent the bisector fit, yielding the range of the AGN slopes. The dashed red lines indicate the range of host slopes determined by Suganuma et al. (2006). The intersection between the host galaxy and AGN slope (red area) gives the host galaxy flux in the respective bands.

NIR $J$ and $K$-bands, which are dominated by the innermost hot dust reacts with delayed response to the AGN continuum.

The time delay between the AGN continuum and dust emission can be estimated by cross-correlation of the optical and NIR light curves yielding the average radius of the innermost dust torus. We correlated both the $B$ - and $K$-band light curves and the $B$ - and $J$-band light curves using the discrete correlation function (DCF, Edelson \& Krolik 1988). The cross-correlation of $B / J$ shows two peaks, one small correlation peak around lag 19 days obtained above the correlation level at $r \geq 0.4 r_{\max }$ and a major peak with a lag of 46 days obtained above the correlation level at $r \geq 0.6 r_{\max }$, as shown in Fig. 16. Similar features can be seen in the cross-correlation of $B / K$, a small correlation peak with a lag of 19 days, a major peak with a lag of 47 days, and an additional third and smallest peak with a lag of 67 days as shown in Fig. 17. The NIR host galaxy corrected light curves show features of similar amplitude and sharpness as the optical host galaxy corrected light curves. In addition, the cross-correlation are at nearly zero level at lag 0 days. One expects that if the dust distribution is spread at different line-of-sight distances, the observed echo will be smeared out in time and the $J$ and $K$ light

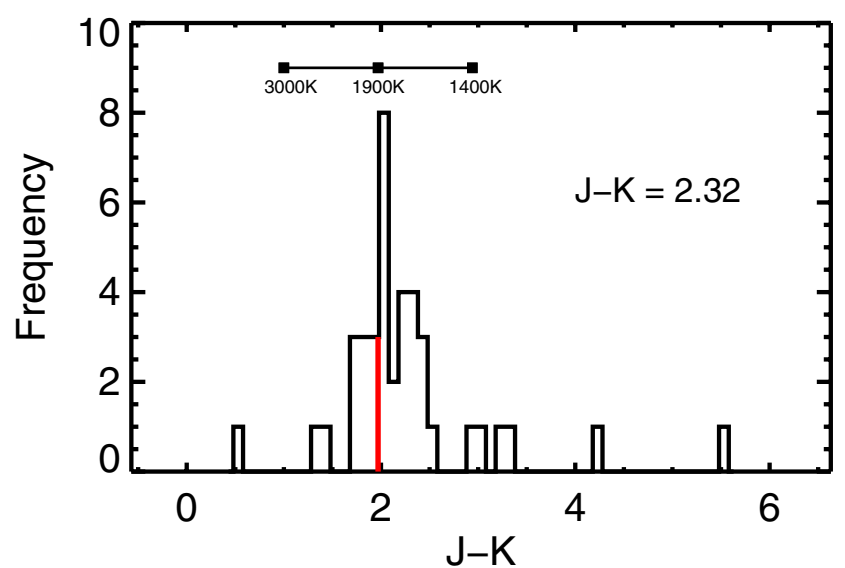

Fig. 15. Distribution of AGN NIR $J-K$ colors of Seyfert galaxies taken from Glass (2004) after host galaxy subtraction. The red vertical solid line correspond to the $J-K$ color of PGC 50427. The filled black squares indicate blackbody color temperatures.

curves will show a smoother variability. Therefore the observed NIR light curves of PGC 50427 argues in favor of a face-on torus geometry.

The uncertainty of the lag time $\tau$ was estimated using the flux randomization and random subset-selection method (FR and RSS, Peterson et al. 2004). The median of this procedure yields $\tau_{\text {cent }}=46.8_{-1.5}^{+1.5}$ days and $\tau_{\text {cent }}=47.8_{-3.2}^{+1.2}$ days for $B / J$ and $B / K$, respectively. Correcting for the time dilation factor, we obtain a rest frame time-delay $\tau_{\text {rest }}=45.7 \pm 1.47$ days and $\tau_{\text {rest }}=46.7 \pm$ 2.15 days for $B / J$ and $B / K$, respectively.

Dust-reverberation studies of Seyfert 1 galaxies have shown that the dust torus size obtained by the cross-correlation of the optical $V$ and $K$-bands is proportional to the square root of the optical luminosity $\tau \propto L^{0.5}$ (Suganuma et al. 2006; Koshida et al. 2014). Figure 18 shows the position of PGC 50427 on the Dust size-luminosity diagram. To obtain the optical $V$-band fluxes we interpolated the AGN $B$ - and $R$-band fluxes.

\section{Summary and conclusions}

We have performed three monitoring campaigns between 2011 and 2014, as well as obtaining a SALT spectrum, of the Seyfert 1 galaxy PGC 50427. We determined the BLR size, the dust torus size, the virial black hole mass and the host-subtracted AGN optical and NIR luminosity. The results are:

1. The cross-correlation of the $\mathrm{H} \alpha$ emission line with the optical continuum during campaign 2011 yields a rest-frame time delay $\tau_{\text {rest }}=19.9 \pm 0.68$ days. During campaign 2014 


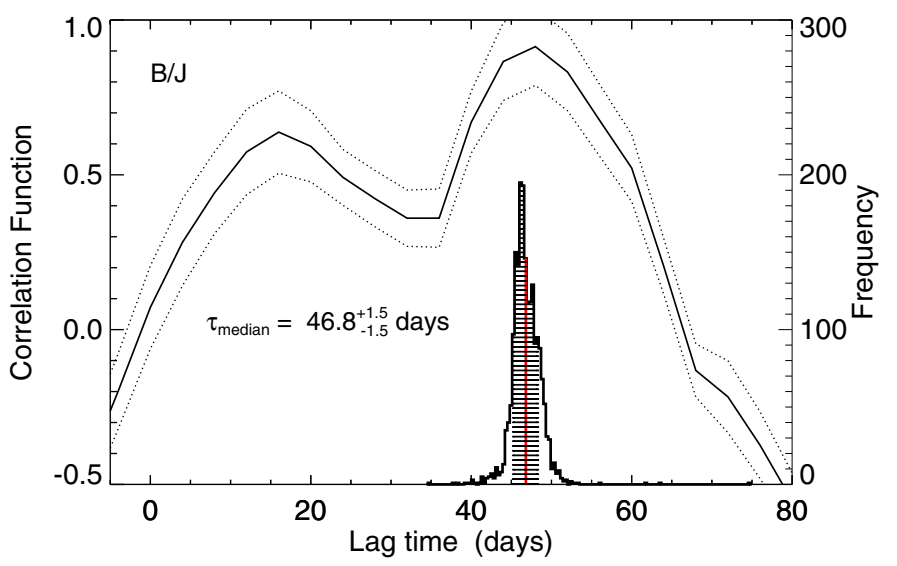

Fig. 16. Cross correlation of $B$ and $J$ light curves. The dotted lines indicate the error range $( \pm 1 \sigma)$ around the cross correlation. The centroid was calculated above the correlation level at $r \geq 0.6 r_{\max }$. The histogram shows the distribution of the centroid lag obtained by cross correlating 2000 flux randomized and randomly selected subset light curves (FR/RSS method). The black shaded area marks the 68\% confidence range used to calculate the errors of the centroid.

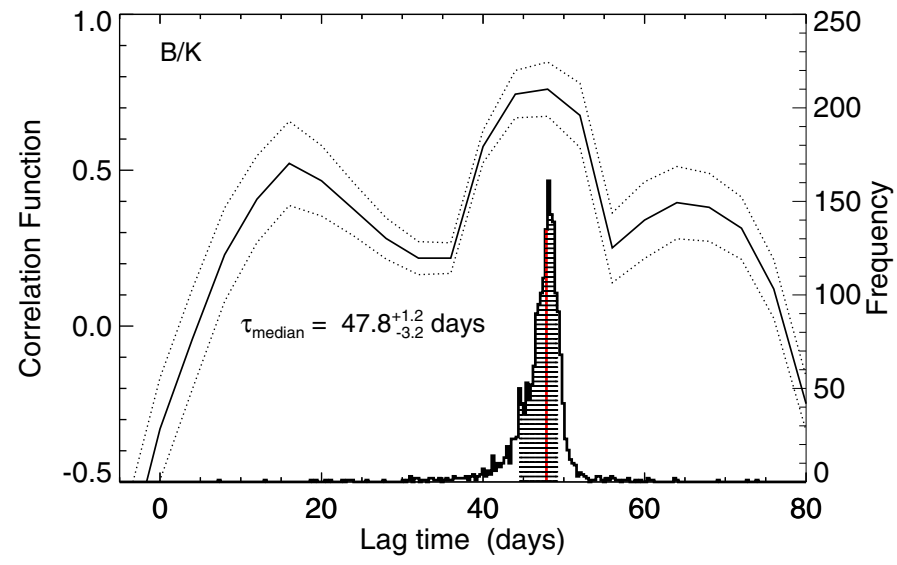

Fig. 17. Same as Fig. 16, but for $B$ and $K$ light curves.

the cross-correlation of the $\mathrm{H} \alpha$ emission line with the optical continuum yields a rest-frame time delay $\tau_{\text {rest }}=18.3 \pm 1.03$.

2. With the velocity dispersion obtained from a single epoch spectrum in 2013, we determine the black hole mass $M_{\mathrm{BH}}=$ $(17 \pm 11) \times 10^{6} M_{\odot}$ assuming a geometrical factor of 5.5.

3 . Using the FVG method we determine the host galaxy subtracted optical AGN luminosity of PGC 50427 at different epochs of observations $L_{\mathrm{AGN}-2011}=(1.18 \pm 0.14) \times$ $10^{43} \mathrm{erg} \mathrm{s}^{-1}, L_{\mathrm{AGN}-2013}=(1.00 \pm 0.12) \times 10^{43} \mathrm{erg} \mathrm{s}^{-1}$, and $L_{\mathrm{AGN}}=(1.22 \pm 0.12) \times 10^{43} \mathrm{erg} \mathrm{s}^{-1}$.

4. From the NIR light curves in 2013 , we determine a lag time of $\tau_{\text {rest }}=45.7 \pm 1.47$ days and $\tau_{\text {rest }}=46.7 \pm 2.15$ days for $B / J$ and $B / K$, respectively. The relatively sharp dust echo observed in the NIR light curves argues in favor of a faceon torus geometry. The infrared lag time is correlated with the optical luminosity according to $R_{\text {dust }} \propto L^{0.5}$ in agreement with previous investigations. The infered inner size for the dust torus in PGC 50427 suggest that the location of the thermal emitting region is located well outside the BLR which support the unified scheme of AGNs.

Acknowledgements. This publication is supported as a project of the NordrheinWestfälische Akademie der Wissenschaften und der Künste in the framework of the academy program by the Federal Republic of Germany

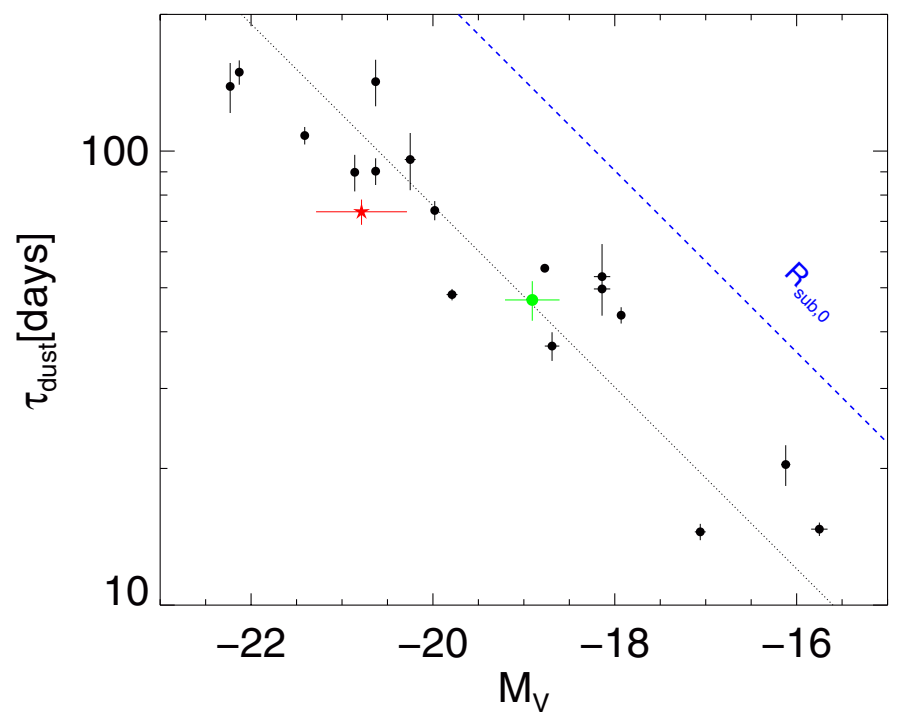

Fig. 18. Lag - luminosity relationship based on the data of Suganuma et al. (2006) and Koshida et al. (2014). The solid line is the best-fit regression from Koshida et al. (2014). PGC 50427 (green dot) lies close to the regression line. The dashed blue line indicates the dust sublimation radius $r_{\mathrm{sub}}$ expected at a given nuclear luminosity $M_{V}$ (from Kishimoto et al. 2007).

and the state Nordrhein-Westfalen, by Deutsche Forschungsgemeinschaft (DFG HA3555/12-1) and by Deutsches Zentrum für Luft-und Raumfahrt (DLR 50 OR 1106). This work has been supported by DFG grant Ko857/32-2. The observations on Cerro Armazones benefitted from the care of the guardians Hector Labra, Gerardo Pino, Roberto Munoz, and Francisco Arraya. Construction of the IRIS infrared camera was supported by the National Science Foundation under grant AST07-04954. Some of the observations reported in this paper were obtained with the Southern African Large Telescope (SALT). We thank Janjenka Szillat for her support regarding the spectral reduction. This research has made use of the NASA/IPAC Extragalactic Database (NED) which is operated by the Jet Propulsion Laboratory, California Institute of Technology, under contract with the National Aeronautics and Space Administration. This research has made use of the SIMBAD database, operated at CDS, Strasbourg, France. We thank our referee Darach Watson for helpful comments and careful review of the manuscript.

\section{References}

Alonso-Herrero, A., Quillen, A. C., Simpson, C., Efstathiou, A., \& Ward, M. J. 2001, AJ, 121, 1369

Antonucci, R. 1993, ARA\&A, 31, 473

Antonucci, R. R. J., \& Miller, J. S. 1985, ApJ, 297, 621

Barvainis, R. 1987, ApJ, 320, 537

Barvainis, R. 1992, ApJ, 400, 502

Bentz, M. C., Denney, K. D., Grier, C. J., et al. 2013, ApJ, 767, 149

Bertin, E. 2006, in Astronomical Data Analysis Software and Systems XV, eds. C. Gabriel, C. Arviset, D. Ponz, \& S. Enrique, ASP Conf. Ser., 351, 112

Bertin, E., Mellier, Y., Radovich, M., et al. 2002, in Astronomical Data Analysis Software and Systems XI, ed. D. A. Bohlender, D. Durand, \& T. H. Handley, ASP Conf. Ser., 281, 228

Blandford, R. D., \& McKee, C. F. 1982, ApJ, 255, 419

Chelouche, D., \& Daniel, E. 2012, ApJ, 747, 62

Chelouche, D., \& Zucker, S. 2013, ApJ, 769, 124

Chelouche, D., Daniel, E., \& Kaspi, S. 2012, ApJ, 750, L43

Cherepashchuk, A. M., \& Lyutyi, V. M. 1973, Astrophys. Lett., 13, 165

Choloniewski, J. 1981, Acta Astron., 31, 293

Czerny, B., \& Hryniewicz, K. 2011, A\&A, 525, L8

Denney, K. D., Peterson, B. M., Dietrich, M., Vestergaard, M., \& Bentz, M. C. 2009, ApJ, 692, 246

Du, P., Hu, C., Lu, K.-X., et al. 2014, ApJ, 782, 45

Edelson, R. A., \& Krolik, J. H. 1988, ApJ, 333, 646

Edri, H., Rafter, S. E., Chelouche, D., Kaspi, S., \& Behar, E. 2012, ApJ, 756, 73

Elitzur, M., \& Shlosman, I. 2006, ApJ, 648, L101

Gaskell, C. M., \& Sparke, L. S. 1986, ApJ, 305, 175

Glass, I. S. 2004, MNRAS, 350, 1049 
Greene, J. E., \& Ho, L. C. 2004, ApJ, 610, 722

Haas, M., Klaas, U., Müller, S. A. H., et al. 2003, A\&A, 402, 87

Haas, M., Müller, S. A. H., Bertoldi, F., et al. 2004, A\&A, 424, 531

Haas, M., Chini, R., Ramolla, M., et al. 2011, A\&A, 535, A73

Haas, M., Hackstein, M., Ramolla, M., et al. 2012, Astron. Nachr., 333, 706

Hodapp, K. W., Chini, R., Reipurth, B., et al. 2010, in SPIE Conf. Ser., 7735

Kabath, P., Erikson, A., Rauer, H., et al. 2009, A\&A, 506, 569

Kawaguchi, T., \& Mori, M. 2010, ApJ, 724, L183

Kawaguchi, T., \& Mori, M. 2011, ApJ, 737, 105

Kishimoto, M., Hönig, S. F., Beckert, T., \& Weigelt, G. 2007, A\&A, 476, 713

Kobayashi, Y., Sato, S., Yamashita, T., Shiba, H., \& Takami, H. 1993, ApJ, 404, 94

Konigl, A., \& Kartje, J. F. 1994, ApJ, 434, 446

Koshida, S., Minezaki, T., Yoshii, Y., et al. 2014, ApJ, 788, 159

Landolt, A. U. 2009, AJ, 137, 4186

Lyutyi, V. M., \& Cherepashchuk, A. M. 1972, Astronomicheskij Tsirkulyar, 688, 1

Minezaki, T., Yoshii, Y., Kobayashi, Y., et al. 2004, ApJ, 600, L35

Mor, R., \& Netzer, H. 2012, MNRAS, 420, 526

Oknyanskij, V. L., Lyuty, V. M., Taranova, O. G., \& Shenavrin, V. I. 1999, Astron. Lett., 25, 483
Patat, F., Moehler, S., O’Brien, K., et al. 2011, A\&A, 527, A91

Peterson, B. M. 1993, PASP, 105, 247

Peterson, B. M., Ferrarese, L., Gilbert, K. M., et al. 2004, ApJ, 613, 682

Pott, J.-U., Malkan, M. A., Elitzur, M., et al. 2010, ApJ, 715, 736

Pozo Nuñez, F., Ramolla, M., Westhues, C., et al. 2012, A\&A, 545, A84

Pozo Nuñez, F., Westhues, C., Ramolla, M., et al. 2013, A\&A, 552, A1

Pozo Nuñez, F., Haas, M., Chini, R., et al. 2014a, A\&A, 561, L8

Pozo Nuñez, F., Haas, M., Ramolla, M., et al. 2014b, A\&A, 568, A36

Ramolla, M., Drass, H., Lemke, R., et al. 2013, Astron. Nachr., 334, 1115

Rodriguez-Pascual, P. M., Santos-Lleo, M., \& Clavel, J. 1989, A\&A, 219, 101

Sakata, Y., Minezaki, T., Yoshii, Y., et al. 2010, ApJ, 711, 461

Schlafly, E. F., \& Finkbeiner, D. P. 2011, ApJ, 737, 103

Siebenmorgen, R., Haas, M., Krügel, E., \& Schulz, B. 2005, A\&A, 436, L5

Suganuma, M., Yoshii, Y., Kobayashi, Y., et al. 2006, ApJ, 639, 46

Tristram, K. R. W., Meisenheimer, K., Jaffe, W., et al. 2007, A\&A, 474, 837

Véron-Cetty, M.-P., \& Véron, P. 2010, A\&A, 518, A10

Vestergaard, M. 2002, ApJ, 571, 733

Winkler, H., Glass, I. S., van Wyk, F., et al. 1992, MNRAS, 257, 659

Wittkowski, M., Kervella, P., Arsenault, R., et al. 2004, A\&A, 418, L39

Woo, J.-H., Treu, T., Malkan, M. A., Ferry, M. A., \& Misch, T. 2007, ApJ, 661, 60 